\title{
ESTRESSE, QUALIDADE DE VIDA E PRESSÃO ARTERIAL DE ESTUDANTES UNIVERSITÁRIOS
}

Luís Henrique Novais

Faculdade de Ciências Médicas de Minas Gerais

Bruno Almeida Rezende

Faculdade de Ciências Médicas de Minas Gerais
Recebido em: 16/03/2020

$1^{\text {a }}$ revisão em: 11/09/2020

$2^{a}$ revisão em: 23/11/2020

Aceito em: 12/05/2021

\section{RESUMO}

A saúde mental de estudantes universitários tem sido foco de investigações, pois apontam para alta prevalência de sintomas de ansiedade e depressão. Este trabalho tem por objetivo avaliar a ocorrência do aumento na prevalência e severidade do estresse, suas consequências somáticas representadas pela elevação dos valores de pressão arterial e sua associação com qualidade de vida em estudantes universitários. Trezentos e dois estudantes foram divididos em áreas acadêmicas e avaliados por meio do Inventário de Estresse de Lipp, Inventário de qualidade de vida WHOQOL-bref, aferição de pressão arterial: no início e no final do semestre letivo. Os resultados apontam para um aumento na prevalência de estresse, aumento dos níveis pressóricos ao final do semestre letivo e piora da qualidade de vida nos momentos estudados. Os resultados indicam uma necessidade de ações para minimizar às condições relacionadas ao surgimento do estresse.

Palavras-chave: estresse; qualidade de vida; estudantes universitários; hipertensão. 


\title{
STRESS, QUALITY OF LIFE AND BLOOD PRESSURE OF UNIVERSITY STUDENTS
}

\begin{abstract}
The mental health of university students has been the focus of investigations, as they point to a high prevalence of symptoms of anxiety and depression. This work aims to evaluate the occurrence of an increase in the prevalence and severity of stress, its somatic consequences represented by the increase in blood pressure values and its association with quality of life in university students. Three hundred and two students were divided into academic areas and evaluated through Lipp's Stress Inventory, WHOQOL-bref quality of life inventory, blood pressure measurement: at the beginning and at the end of the academic semester. The results point to an increase in the prevalence of stress, an increase in blood pressure levels at the end of the school semester and a worsening of the quality of life in the studied moments. The results indicate a need for actions to minimize conditions related to the emergence of stress.
\end{abstract}

Keywords: stress; quality of life; university students; hypertension.

\section{ESTRÉS, CALIDAD DE VIDA Y PRESIÓN ARTERIAL DE ESTUDIANTES UNIVERSITARIOS}

\section{RESUMEN}

La salud mental de los estudiantes universitarios ha sido el foco de investigaciones, ya que apuntan a una alta prevalencia de síntomas de ansiedad y depresión. Este trabajo tiene como objetivo evaluar la ocurrencia de un aumento en la prevalencia y severidad del estrés, sus consecuencias somáticas representadas por el aumento de los valores de presión arterial y su asociación con la calidad de vida en estudiantes universitarios. Trescientos dos estudiantes fueron divididos en áreas académicas y evaluados a través del Inventario de Estrés de Lipp, inventario de calidad de vida WHOQOL-bref, medición de la presión arterial: al inicio y al final del semestre académico. Los resultados apuntan a un aumento de la prevalencia de estrés, un aumento de los niveles de presión arterial al final del semestre escolar y un empeoramiento de la calidad de vida en los momentos estudiados. Los resultados indican la necesidad de acciones para minimizar las condiciones relacionadas con la aparición del estrés.

Palabras clave: estrés; calidad de vida; estudiantes universitarios; hipertensión. 


\section{INTRODUÇÃO}

O organismo humano é o resultado de bilhões de anos de evolução e constantes adaptações ao ambiente. O conceito de adaptação de acordo com Stearns e Hoekstra (2003) é uma variação hereditária do organismo humano através do processo reprodutivo. Para estes autores é o processo reprodutivo que possibilita aos fenótipos atuarem nos genótipos, permitindo gerar seres mais adaptativos para sua sobrevivência e de sua espécie. O estresse, como apontam os autores, é um processo adaptativo que permite ao organismo interagir com o seu meio ambiente e garantir sua sobrevivência.

A capacidade de adaptação ao ambiente ocorre por modificações nos processos fisiológicos do organismo. Estas alterações são eliciadas a partir do efeito de estressores que produzem alterações fisiológicas nos órgãos internos do organismo humano e por sua vez permitem que estes respondam de forma adaptativa ao ambiente (Agorastos, Pervanidou, Chrousos, \& Baker, 2019). São os núcleos neuronais presentes no locus coeruleus, localizados na região cinzenta do tronco encefálico, os responsáveis pelos impulsos necessários para a ativação hormonal. Os impulsos axonais provenientes do locus coeruleus são por sua vez distribuídos para todo o córtex cerebral, diencéfalo e hipotálamo. No hipotálamo estes sinais estimulam a hipófise na produção dos hormônios precursores dos hormônios adrenocorticais. Com a ativação da glândula adrenal, ocorrerá a produção de catecolaminas e cortisol. Esta produção e sua distribuição no organismo humano provocará a alteração do metabolismo de diversos órgãos, permitindo a adaptação frente as alterações do meio ambiente, através de respostas somáticas (Valentino \& Van Bockstaele, 2008; Wood \& Valentino, 2017). Assim, diante de um estímulo do ambiente, o organismo reagirá de maneira imediata através de respostas somáticas a qualquer tipo de risco ou ameaça, como acontece durante o estado de estresse. O organismo aciona um estado de alerta e se coloca em prontidão para emitir uma resposta de luta ou esquiva frente à situação de perigo (Lipp, 1984). Esta resposta leva o organismo a alterações imediatas como vasodilatação muscular e cerebral, aumento do débito cardíaco e dilatação pupilar (Lipp, 1984). Esta suprarregulação hormonal pode gerar tanto respostas imediatas como o aumento da sudorese, tensão muscular, taquicardia, hiperatividade, náuseas quanto respostas mais crônicas como gastrite crônica, alterações dermatológicas e elevação dos níveis de pressão arterial sistêmica (Yaribeygi, Panahi, Sahraei, Johnston, \& Sahebkar, 2017). Neste último caso, esta relação já havia sido observada desde 1945 por Graham que mostrou que soldados expostos as batalhas mantinham elevação de níveis pressóricos mesmo depois de várias semanas após o combate (Graham, 1945). Desde então, vários trabalhos já apontaram a exposição a situações estressoras como fator relacionado ao aumento dos níveis pressóricos, podendo desencadear hipertensão arterial (Matthews et al., 2004; Sheffield, Smith, Carroll, Shipley, \& Marmot, 1997).

O estresse pode afetar a saúde, a qualidade de vida e a sensação de bem-estar como um todo, tornando necessário sua identificação e quantificação (Lipp,1984; 
Sadir, Bignotto, \& Lipp, 2010). A partir de uma correta identificação e avaliação de sua gravidade, intervenções farmacológicas e/ou psicoterapêuticas podem ser feitas no sentido de minimizar sua gravidade, assim como suas repercussões psicossomáticas. Atualmente, existem ferramentas classificatórias que, a partir de avaliações fisiológicas, cognitivas, comportamentais e sociais podem ser facilmente empregadas na tentativa da identificação de diferentes fases adaptativas do estresse ao qual um indivíduo está submetido, possibilitando o desenvolvimento de ferramentas para a avaliação do nível de estresse.

O estresse foi postulado por Hans Selye como uma resposta orgânica, não específica, baseada no conceito da síndrome de adaptação geral (SGA) apresentada em três diferentes fases: alerta, resistência e exaustão (Selye, 1959). A fase de alerta ou alarme se caracteriza por manifestações agudas que levam a uma adaptação do organismo (estresse) diante modificações do ambiente, buscando a homeostasia por meio da liberação de adrenalina para o organismo através da corrente sanguínea. Na fase de resistência, as manifestações agudas desaparecem através da manutenção do equilíbrio interno adaptativo na continuidade dos agentes estressores. Por fim, a fase de exaustão ocorre quando não ocorre um retorno das reações adaptativas das fases alerta e resistência, o que consequentemente provocará o surgimento de respostas crônicas no organismo. Haverá uma quebra na resistência do organismo quando houver continuidade dos agentes estressores, sobrecarregando as funções adaptativas na qual não ocorrerão respostas satisfatórias e homeostáticas diante dos estímulos do ambiente (Rossetti et al., 2008). Segundo Selye, o estresse pode ser encontrado em qualquer uma das fases citadas, ainda que as manifestações sejam diferentes ao longo do tempo. Não é necessário que as três fases se desenvolvam para haver o registro da síndrome, já que somente o estresse mais grave leva à fase de exaustão (Selye, 1959).

Os estudos de Selye serviram de base para o desenvolvimento do inventário de avaliação de estresse de LIPP. Este inventário investiga as três fases do estresse, suas manifestações e seus aspectos físicos, psicológicos e sociais através de perguntas que se referem ao sistema de autoconceituação ou autopercepção do investigando (Lipp \& Guevara, 1994).

Na literatura científica é demonstrado que o estresse está intimamente relacionado à qualidade de vida dos indivíduos. Ao se analisar as fases do estresse parece racional avaliar todo o contexto de qualidade de vida em que o indivíduo está inserido, uma vez que as características psicológicas e sociais possuem forte impacto na exposição aos agentes estressores e suas respostas fisiológicas (Bujang Musa, Liu, Chew, Lim, \& Morad, 2015; Sadir et all, 2010).

Apesar da definição não ser tão precisa em parâmetros, o conceito de qualidade de vida abrange os aspectos físicos, psicológicos e sociais. Ter qualidade de vida é estar em condições satisfatórias de usufruir tais aspectos. Para avaliar a qualidade de vida da população, a Organização Mundial de Saúde (OMS) desenvolveu em 
1990 uma ferramenta que recebeu a designação WHOQOL-100 (Group, 1995). Trata-se de um inventário que consiste em 100 questões que abordam os aspectos da qualidade de vida das pessoas. As repostas são elaboradas através da autopercepção e autocompreensão nos domínios: físico, psicológico, social e ambiental. Para facilitar a compreensão e respostas do inventário, foi utilizada a escala de Likert. Este inventário foi adaptado posteriormente para diferentes contextos e culturas para as diversas populações ao redor do mundo e seus aspectos e singularidades; versões simplificadas destes inventários foram validadas visando tornar mais prática a avaliação em diversos estudos (Bujang et al., 2015; Damião Neto, Lucchetti, da Silva Ezequiel, \& Lucchetti, 2019). Posteriormente este inventário passou por algumas reformulações que permitiram a diminuição do tempo de aplicação e uma maior efetividade no tratamento dos dados obtidos. Esta nova atualização do WHOQOL recebeu o nome de WHOQOL-Bref.

A vida acadêmica do discente está marcada por período de anos. Este período pode variar, dependendo do curso em questão. Grande parte dos discentes precisam adaptar sua vida, sua rotina diária para adequar à vida acadêmica. Esta adequação pode levar a uma sobrecarga física, psicológica e social do discente. É comum a manifestação sintomática do estresse diante o semestre letivo. O próprio corpo discente afirma que há uma enorme diferença no rendimento por parte dos acadêmicos se levarmos em consideração o período do semestre letivo em curso (Cardoso, Gomes, Junior, \& Silva, 2019; Saleh, Camart, \& Romo, 2017). No início do semestre letivo, os alunos voltam de períodos de férias e ainda não possuem muitas atribuições acadêmicas. Já ao final do semestre, o discente se depara com um grande volume de atribuições, trabalhos, apresentações, eventos acadêmicos e provas que são claramente agentes estressores. Diante desta problemática, este trabalho tem por objetivo avaliar o nível de estresse e qualidade de vida de alunos universitários de um centro universitário privado em fase inicial e final do semestre letivo. A pressão arterial destes indivíduos também foi monitorada a fim de se avaliar desenvolvimento de alterações crônicas relacionadas ao possível incremento do nível de estresse.

\section{METODOLOGIA}

Trata-se de um estudo de coorte longitudinal a partir de uma amostra de conveniência.

\section{PARTICIPANTES}

Participaram deste estudo 302 estudantes universitários com idade superior a 18 anos de ambos os sexos, matriculados em um Centro Universitário privado localizada na cidade de Divinópolis no estado de Minas Gerais entre o período de fevereiro e julho de 2019. Os cursos foram distribuídos de acordo com suas áreas de concentração, totalizando 5 áreas técnicas que formaram os grupos do estudo: ciências humanas, ciências exatas, ciências biológicas, ciências médicas e tecnólogos. Os estudantes pertencentes a cada grupo foram selecionados 
aleatoriamente. A distribuição dos participantes levou em conta a proporção de matrículas em cada área para a elaboração do cálculo amostral. Em relação aos cursos pertencentes a cada área na instituição avaliada, a área de ciências humanas compreende os cursos de psicologia, comunicação social, administração e direito. A área de ciências exatas compreende os cursos de engenharias, contabilidade e sistema de informação. A área de ciências biológicas compreende o curso de farmácia e a área de ciências médicas compreende o curso de enfermagem. A área de tecnólogos compreende diversos cursos superiores de tecnologia.

Por haver uma grande discrepância entre o número de alunos por área na instituição onde foi realizada a pesquisa, foram incluídos no estudo uma representatividade de cerca de $4,5 \%$ do número total de alunos por área (Tabela 1). Apenas os estudantes que cumpriram todas as etapas da pesquisa foram incluídos no estudo.

Tabela 1.

Representatividade dos participantes da pesquisa.

\begin{tabular}{cccc}
\hline ÁREA & $\begin{array}{c}\text { PARTICIPANTES } \\
(\%)\end{array}$ & AMOSTRA & REPRESENTATIVIDADE \\
Humanas & $3.218(47,5 \%)$ & 143 & $4,4 \%$ \\
Exatas & $2.748(40,6 \%)$ & 123 & $4,47 \%$ \\
Biológicas & $248(3,7 \%)$ & 11 & $4,43 \%$ \\
Médicas & $315(4,6 \%)$ & 14 & $4,44 \%$ \\
Tecnólogos & $241(3,6 \%)$ & 11 & $4,56 \%$ \\
Total & $6.770(100 \%)$ & 302 & $4,46 \%$ \\
\hline
\end{tabular}

Representatividade dos participantes da pesquisa por área técnica na instituição de ensino de acordo com o número de matrículas.

\section{INSTRUMENTOS}

Inventário de Estresse de Lipp ISSL. Este instrumento se baseia na avaliação dos sintomas físicos e psicológicos do sujeito adulto para fornecer a mensuração objetiva dos sintomas de estresse. O Instrumento é composto por três quadros referentes às fases do estresse. O primeiro quadro compreende quinze itens referentes aos sintomas físicos ou psicológicos que o indivíduo experimentou nas últimas 24 horas. O segundo é composto de dez sintomas físicos e cinco psicológicos experimentados na última semana. O terceiro quadro compreende doze sintomas físicos e onze psicológicos que se referem a sintomas experimentados no último mês. Este instrumento busca identificar a sintomatologia, o tipo de sintoma e classificar o nível de estresse de acordo com a 
fase vivenciada pelo participante da pesquisa (alerta, resistência ou exaustão) (Lipp \& Guevara, 1994; Rossetti et al., 2008).

Inventário de qualidade de vida WHOQOL-bref. Visando dinamizar o inventário WHOQOL-100, foi elaborada a versão simplificada deste questionário, recebendo a designação WHOQOL-bref (Kluthcovsky \& Kluthcovsky, 2009). Esta versão consiste em 24 questões distribuídas em 4 domínios: físico, psicológico, relações sociais e meio ambiente. A versão brasileira do WHOQOL-bref foi adaptada e validada pelo núcleo de pós-graduação em psiquiatria da Universidade Federal do Rio Grande do Sul (Kluthcovsky \& Kluthcovsky, 2009).

Aferição da Pressão Arterial Sistêmica. A aferição da pressão arterial sistêmica se deu através do uso de um esfigmomanometro digital, modelo Microlife BP A100, número de série 331606491. Este aparelho foi validado para pesquisa de acordo com critérios estabelecidos pela European Society of Hypertension (O'Brien et al., 2010).

\section{PROCEDIMENTOS}

Os estudantes foram aleatoriamente selecionados a participarem da pesquisa através de recrutamento na instituição no período correspondente às duas primeiras semanas de aula do semestre letivo institucional. Após orientações e assinatura do Termo de Consentimento Livre e Esclarecido (TCLE), os mesmos receberam o Inventário de Estresse de Lipp ISSL e o Inventário de qualidade de vida WHOQOL-bref para preenchimento. Após o preenchimento dos inventários, a pressão arterial dos estudantes foi aferida.

As aferições foram realizadas a partir de três medidas consecutivas com o intervalo de 1 minuto entre elas. A primeira aferição foi descartada com o objetivo de evitar qualquer reação ou resposta adversa adaptativa por parte do participante. A média das duas últimas medidas foi considerada como sendo a pressão arterial real. Caso as pressões sistólicas e/ou diastólicas obtidas apresentassem uma diferença maior que $4 \mathrm{mmHg}$ entre as medições no mesmo participante, eram realizadas novas medidas até que se obtivessem medidas com diferença inferior. Todos estes critérios para a aferição da PA foram seguidos de acordo com o que foi preconizado pela European Society of Hypertension (Bittencourt et al., 1971). Este protocolo foi novamente aplicado para os mesmos estudantes no período correspondente às duas últimas semanas do semestre letivo da instituição participante. Desta forma pudemos fazer a comparação dos resultados nas duas etapas descritas como Etapa 1 e Etapa 2. Foram inseridos no estudo apenas os estudantes que concretizaram as duas etapas de avaliação.

\section{ANÁLISE ESTATÍSTICA}

As variáveis qualitativas foram apresentadas como frequências absolutas e relativas, e as quantitativas como média \pm desvio-padrão da média e a mediana. As variáveis quantitativas foram submetidas ao teste de normalidade de Shapiro- 
Wilk. A comparação de variáveis quantitativas entre dois grupos foi realizada via teste de Wilcoxon ou $t$-Student para amostras pareadas. Para a avaliação da associação entre variáveis qualitativas nas duas etapas utilizou-se o teste quiquadrado de McNemar ou modelo logístico binário. As análises foram desenvolvidas no programa gratuito $R$ versão 3.5 .1 e foi considerado significativo $p<0,05$.

\section{ASPECTOS ÉTICOS}

Todos os participantes do estudo assinaram o TCLE após a explicação prévia do projeto, leitura dos termos e esclarecimentos de possíveis dúvidas a respeito da pesquisa. Este projeto de pesquisa foi aprovado pelo comitê de Ética em pesquisa da Faculdade de Ciências Médicas de Minas Gerais - FELUMA sob o parecer número: 2.486.657, data de 06 de fevereiro de 2018. Todos os procedimentos envolvidos neste estudo estão de acordo com a Declaração de Helsinki de 1975, atualizada em 2013.

\section{RESULTADOS}

Dos 302 estudantes avaliados, 83 (27,48\%) eram do sexo masculino e 219 (72,52\%) do sexo feminino. Em todas as áreas técnicas avaliadas foi constatado um aumento no número de indivíduos com estresse entre o início e o final do semestre letivo (Tabela 2).

Para os estudantes pertencentes às áreas de ciências biológicas e tecnólogos, esta variação se mostrou significativa para ciências biológicas $(p<0,001)$ e tecnólogos $(p<0,041)$, respectivamente. Ao serem avaliadas as diferentes fases do estresse nas duas etapas estudadas, observamos que os estudantes pertencentes às áreas de humanas mostraram evolução do nível de estresse para fases de maior gravidade, diminuindo a proporção de estudantes na fase de alerta e aumentando sua proporção na fase de exaustão ao final do semestre letivo (Tabela 2; $p=0,030$ ). Para os universitários das áreas de ciências médicas e tecnólogos, apesar de observarmos um aumento no número de indivíduos com estresse, não observamos modificação na fase do estresse entre as etapas estudadas $(p=1,000)$. Para a área de exatas, apesar de observarmos uma maior proporção de indivíduos em fases mais avançadas de estresse, não foi observada diferença estatística significativa entre as etapas 1 e 2.

Tabela 2.

Avaliação do estresse segundo etapa da avaliação e área técnica.

\begin{tabular}{lccc}
\multicolumn{1}{c}{ VARIÁVEIS } & ETAPA 1 & ETAPA 2 & P-VALOR \\
\hline $\begin{array}{l}\text { Estudantes com } \\
\text { estresse }\end{array}$ & & & \\
$\quad$ Biológicas & $3(27,3 \%)$ & $8(72,7 \%)$ & $0,074^{\mathrm{M}}$ \\
\hline
\end{tabular}




\begin{tabular}{|c|c|c|c|}
\hline Exatas & $63(51,2 \%)$ & $85(69,1 \%)$ & $<0,001^{\mathrm{M}}$ \\
\hline Humanas & $101(70,6 \%)$ & 109 (76,2\%) & $0,216^{M}$ \\
\hline Médicas & $6(42,9 \%)$ & $9(64,3 \%)$ & $0,248^{M}$ \\
\hline Tecnólogos & $3(27,3 \%)$ & $9(81,8 \%)$ & $\mathbf{0 , 0 4 1 ^ { \mathrm { M } }}$ \\
\hline Total & $176(58,3 \%)$ & $220(72,8 \%)$ & $<0,001^{\mathrm{M}}$ \\
\hline \multicolumn{4}{|l|}{ Fases do estresse } \\
\hline Biológicas & & & $0,223^{\mathrm{L}}$ \\
\hline Alerta & $1(33,3 \%)$ & - & \\
\hline Resistência & $2(66,7 \%)$ & 6 (75\%) & \\
\hline Exaustão & - & 2 (25\%) & \\
\hline Exatas & & & $0,733^{\mathrm{L}}$ \\
\hline Alerta & 1 (1,6\%) & $2(2,4 \%)$ & \\
\hline Resistência & 49 (73\%) & $57(67,1 \%)$ & \\
\hline Exaustão & $16(25,4 \%)$ & $26(30,6 \%)$ & \\
\hline Humanas & & & $0,030^{L}$ \\
\hline Alerta & $2(2 \%)$ & - & \\
\hline Resistência & $75(75,8 \%)$ & $69(63,3 \%)$ & \\
\hline Exaustão & $22(22,2 \%)$ & $40(36,7 \%)$ & \\
\hline Médicas & & & $1,000^{\mathrm{L}}$ \\
\hline Alerta & - & - & \\
\hline Resistência & $4(66,7 \%)$ & $6(66,7 \%)$ & \\
\hline Exaustão & $2(33,3 \%)$ & $3(33,3 \%)$ & \\
\hline Tecnólogos & & & $1,000^{L}$ \\
\hline Alerta & - & - & \\
\hline Resistência & $3(100 \%)$ & $9(100 \%)$ & \\
\hline Exaustão & - & - & \\
\hline Total & & & $0,080^{L}$ \\
\hline Alerta & $4(2,3 \%)$ & $2(0,9 \%)$ & \\
\hline Resistência & $132(74,7 \%)$ & $147(66,8 \%)$ & \\
\hline Exaustão & 40 (23\%) & $71(32,3 \%)$ & \\
\hline
\end{tabular}

Nota:p-valores: ${ }^{\mathrm{M}}$ teste qui-quadrado de McNemar, ${ }^{\mathrm{L}}$ modelo logístico binário 
Ao se avaliar os valores pressóricos (Tabela 3), observamos um aumento tanto na pressão arterial sistólica quanto na diastólica entre as duas etapas do estudo para as áreas de ciências humanas $(p<0,001)$, exatas $(p<0,001)$, e tecnólogos $(p=0,001$ e $0,002)$. A área de ciências médicas mostrou aumento somente dos valores pressóricos sistólicos $(p=0,016)$. Já a área de ciências biológicas não mostrou alteração pressórica significativa entre as diferentes etapas do estudo.

\section{Tabela 3.}

Avaliação da pressão arterial segundo etapa da avaliação e área técnica.

\begin{tabular}{lccc}
\hline VARIÁVEIS & ETAPA 1 & ETAPA 2 & P-VAL \\
Pressão sistólica & & & \\
Biológicas & $114,2 \pm 9,3(114,8)$ & $121 \pm 14,9(120)$ & $0,107^{\top}$ \\
Exatas & $112 \pm 11,1(111)$ & $115,8 \pm 10,9(117)$ & $<\mathbf{0 , 0 0 1}$ \\
Humanas & $111,4 \pm 14,1(110,5)$ & $114,1 \pm 12,9(114)$ & $<\mathbf{0 , 0 0 1}$ \\
Médicas & $111,8 \pm 14,1(107,5)$ & $115,1 \pm 16,3(110)$ & $\mathbf{0 , 0 1 6}$ \\
Tecnólogos & $119,9 \pm 9,5(120)$ & $125,7 \pm 9,7(124)$ & $\mathbf{0 , 0 0 1}$
\end{tabular}

Pressão

diastólica

$\begin{array}{llll}\text { Biológicas } & 75,1 \pm 6,9(77,5) & 77,3 \pm 8,1(80,3) & 0,282^{\top} \\ \text { Exatas } & 71,6 \pm 9,2(68,5) & 74,1 \pm 8,4(73,5) & <\mathbf{0 , 0 0 1} \\ \text { Humanas } & 73,2 \pm 9,5(72,5) & 76 \pm 9,6(75) & <\mathbf{0 , 0 0 1} \\ \text { Médicas } & 72,5 \pm 9(71,5) & 74,5 \pm 8,1(75) & 0,136^{\top} \\ \text { Tecnólogos } & 76,5 \pm 6,3(78,5) & 81,2 \pm 4,5(82,5) & \mathbf{0 , 0 0 2}\end{array}$

Dados apresentados como média \pm desvio-padrão (mediana). Os $p$-valores referem-se ao teste $t$-Student quando apresentam o símbolo ${ }^{\top}$ e Wilcoxon para amostras pareadas quando não apresentam.

A Tabela 4 apresenta a variação entre os escores dos domínios avaliados no instrumento WHOQOL-bref em ambas as etapas do estudo para as áreas técnicas avaliadas. As duas primeiras variáveis da Tabela 4 apresentam a auto percepção dos universitários em relação a sua qualidade de vida e saúde. Podemos observar que somente os estudantes da área tecnólogos auto referiram uma piora em sua qualidade de vida e satisfação com a saúde ao final do semestre letivo ( $p=0,037$ e 0,048 para ambos os domínios). Há claramente uma tendência de piora entre ambas as etapas do estudo para a maioria dos domínios avaliados pelo instrumento WHOQOL-bref. Estes valores se mostraram significativos no domínio físico e psicológico para as áreas de ciências exatas $(p=0,018$ e 0,006) e humanas $(p<0,001)$. O domínio correspondente ao meio ambiente também mostrou piora 
significativa ao final do semestre letivo para as áreas de ciências biológicas e exatas $(p=0,022$ e 0,035$)$.

\section{Tabela 4.}

Avaliação da qualidade de vida segundo etapa da avaliação e área técnica.

\begin{tabular}{llll}
\multicolumn{1}{c}{ Variáveis } & Etapa 1 & Etapa 2 & P-valo \\
\hline Qualidade de vida referida & & & * \\
Biológicas & $79,5 \pm 11,1(75)$ & $79,5 \pm 11,1(75)$ & \\
Exatas & $72,2 \pm 18,2(75)$ & $70,7 \pm 18(75)$ & 0,380 \\
Humanas & $73,1 \pm 17,1(75)$ & $71,2 \pm 17,1(75)$ & 0,252 \\
Médicas & $80,4 \pm 14,5(75)$ & $75 \pm 13,9(75)$ & 0,149 \\
Tecnólogos & $70,5 \pm 18,8(75)$ & $59,1 \pm 16,9(50)$ & $\mathbf{0 , 0 3 7}$ \\
Satisfação com a Saúde & & & \\
Biológicas & $84,1 \pm 12,6(75)$ & $77,3 \pm 17,5(75)$ & 0,233 \\
Exatas & $65,4 \pm 23(75)$ & $67,1 \pm 19,3(75)$ & 0,508 \\
Humanas & $68,4 \pm 20,8(75)$ & $68,5 \pm 19,4(75)$ & 0,935 \\
Médicas & $80,4 \pm 17,5(75)$ & $73,2 \pm 24,9(75)$ & 0,240 \\
Tecnólogos & $65,9 \pm 12,6(75)$ & $52,3 \pm 23,6(50)$ & $\mathbf{0 , 0 4 8}$
\end{tabular}

Domínio físico

$\begin{array}{llll}\text { Biológicas } & 58,1 \pm 14,1(60,7) & 55,2 \pm 14,7(57,1) & 0,203^{t} \\ \text { Exatas } & 62 \pm 24,1(60,7) & 58,7 \pm 14,8(57,1) & \mathbf{0 , 0 1 8} \\ \text { Humanas } & 69,5 \pm 27,2(67,9) & 65,8 \pm 30,5(60,7) & <\mathbf{0 , 0 0 1} \\ \text { Médicas } & 67,1 \pm 19,4(71,4) & 66,6 \pm 20,4(71,4) & 0,753^{t} \\ \text { Tecnólogos } & 56,2 \pm 13,3(60,7) & 65,6 \pm 31,2(64,3) & 0,057\end{array}$

Domínio psicológico

$\begin{array}{llll}\text { Biológicas } & 84,1 \pm 65,8(66,7) & 64,4 \pm 12,8(66,7) & 0,234 \\ \text { Exatas } & 60 \pm 14(62,5) & 58,5 \pm 16,9(58,3) & \mathbf{0 , 0 0 6} \\ \text { Humanas } & 64,3 \pm 22,8(66,7) & 39,8 \pm 31,3(50) & <\mathbf{0 , 0 0 1} \\ \text { Médicas } & 69,9 \pm 17(72,9) & 69,6 \pm 17(72,9) & 0,623 \\ \text { Tecnólogos } & 49,2 \pm 15,8(54,2) & 47,7 \pm 18,4(50) & 0,420^{t}\end{array}$

Relações sociais

$\begin{array}{llll}\text { Biológicas } & 80,3 \pm 14,6(83,3) & 75,8 \pm 18,4(83,3) & 0,140^{t} \\ \text { Exatas } & 65,8 \pm 21,3(66,7) & 64,3 \pm 19,5(66,7) & 0,233\end{array}$




\begin{tabular}{llll}
\hline Humanas & $69 \pm 15,7(66,7)$ & $68,4 \pm 18,6(75)$ & 0,795 \\
Médicas & $74,4 \pm 24,3(75)$ & $88,7 \pm 64,8(75)$ & 1,000 \\
Tecnólogos & $59,8 \pm 24,4(58,3)$ & $78 \pm 75,1(58,3)$ & 1,000 \\
Meio ambiente & & & \\
Biológicas & $77,6 \pm 50,2(68,8)$ & $60,5 \pm 11,5(59,4)$ & $\mathbf{0 , 0 2 2}$ \\
Exatas & $60,1 \pm 14,8(59,4)$ & $58,6 \pm 13,2(59,4)$ & $\mathbf{0 , 0 3 5}$ \\
Humanas & $60,4 \pm 12,8(59,4)$ & $61,6 \pm 21,3(59,4)$ & 0,570 \\
Médicas & $65,9 \pm 12,5(64)$ & $62,1 \pm 11,9(64,1)$ & $\mathbf{0 , 0 2 9}$ \\
Tecnólogos & $50,6 \pm 18,3(53,1)$ & $47,4 \pm 20,4(46,9)$ & $\mathbf{0 , 1 9 9}$
\end{tabular}

Dados apresentados como média \pm desvio-padrão (mediana). Os $p$-valores referem-se ao teste $t$-Student quando apresentam o símbolo ${ }^{\top} e$ Wilcoxon para amostras pareadas quando não apresentam. *Os valores de QV foram rigorosamente iguais nas duas etapas.

\section{DISCUSSÃO}

O presente trabalho ressalta a necessidade de atenção destinada aos estudantes universitários, visto que estes, durante o curso de sua graduação, passam por intensa carga emocional e um aumento no estresse ocupacional. Nossos resultados claramente apontam para um aumento na prevalência de estresse e piora do nível de estresse ao longo do semestre letivo com evidentes manifestações somáticas, aqui representadas por uma piora dos níveis pressóricos dos estudantes ao final do semestre letivo. Há na literatura muitos estudos transversais apontando um elevado nível de estresse e piora da qualidade de vida em estudantes universitários. No entanto, há uma escassez de estudos com abordagem longitudinal nesta população. Nosso trabalho propôs avaliar os estudantes em duas etapas distintas do semestre letivo para mostrar que o sistema universitário leva a uma sobrecarga dos estudantes ao longo do semestre e isto poderia contribuir para a geração de transtornos emocionais que poderiam causar ansiedade e estados depressivos. Estima-se que 20\% dos estudantes universitários apresentam algum tipo de transtorno psiquiátrico durante o período acadêmico (Adewuya, Ola, Aloba, Mapayi, \& Oginni, 2006; Ibrahim, Kelly, Adams, \& Glazebrook, 2013). Atualmente, vivemos um período de grande discussão a respeito da saúde mental de estudantes universitários. Os índices de suicídio nesta população têm crescido e o assunto tem ganhado bastante repercussão (Mortier et al., 2018; Russell et al., 2019).

Vários trabalhos relacionam o aparecimento do estresse ao desenvolvimento de sintomas somáticos como elevação da pressão arterial (Lipp, Pereira, Justo, \& de Matos, 2006; Matthews et al., 2004). No nosso estudo, todos os grupos estudados mostraram aumento do número de indivíduos com estresse ao final do semestre letivo. No entanto, os grupos de ciências biológicas, humanas e médicas não mostraram significância estatística para esta variável apesar do aumento em 
valores absolutos. Verificamos também que existe uma progressão do nível de estresse para fases mais críticas nos universitários do grupo de ciências humanas, mesmo este grupo não apresentando aumento significativo de estudantes com estresse ao final do semestre letivo. Além disso a elevação pressórica, que pode ser correlacionada ao aumento do nível e na prevalência de estresse (Lipp et al., 2006; Matthews et al., 2004; Sheffield et al., 1997), em quase todos os grupos estudados sugere relação com os dados de aumento na prevalência do estresse obtidos no estudo. Para os grupos de ciências biológicas e médicas, acreditamos que os dados não tenham sido estatisticamente significantes devido à baixa amostragem, sendo este considerado um fator limitante do estudo.

Os dados observados na Tabela 3, indicam a dimensão do ser humano através da sua autopercepção e da leitura corrente que o mesmo faz de sua saúde física, psicológica e social. O corpo discente detém-se de um período do seu dia para a sua formação. Fora do âmbito escolar existem uma infinidade de situações vivenciadas por parte do aluno. Assim, estes fatores podem estar inferidos em respostas estressoras. O inventário de qualidade de vida (WHOQOL-Bref) pode através de seu aspecto biopsicossocial criar parâmetros avaliativos e indicativos do ser humano e a sua relação com os seus aspectos biológicos, psicológicos, sociais e ambientais. Analisando estes parâmetros, verificamos que os estudantes pertencentes ao grupo de ciências exatas, apesar de não autorreferirem piora da qualidade de vida e saúde, foram os que mostraram maior piora dos índices de qualidade de vida ao longo do semestre letivo, com exceção do domínio de relações sociais, que se mostrou inalterado. Este mesmo grupo também mostrou aumento no número de indivíduos com estresse e aumento dos níveis pressóricos ao final do semestre letivo. Alguns trabalhos também têm correlacionado uma piora da qualidade de vida ao aumento do nível de estresse (Ribeiro et al., 2017; Meyer, Guimarães, Machado, \& Parcias, 2012; Sadir, Bignotto \& Lipp, 2010).

No entanto nosso estudo também apontou elevação no número de estudantes com estresse e alterações pressóricas, porém sem piora da qualidade de vida, como por exemplo para os alunos da área de tecnólogos. Este grupo, ao contrário dos estudantes da área de ciências exatas, autorreferiram piora da qualidade de vida e saúde, mas não apontaram piora significativa em nenhum dos domínios de qualidade de vida. Assim, não podemos sugerir para estes grupos, a partir de nosso trabalho, que o aumento na prevalência de estresse ao final do semestre letivo esteja relacionado a uma piora da qualidade de vida. Este tema já foi estudado também por outros autores como Meyer e colaboradores (2012) que investigaram a relação entre estresse ocupacional e qualidade de vida de estudantes de medicina e mostraram que os estudantes de medicina apresentaram melhora na qualidade de vida, mesmo estando expostos a elevados níveis de estresse (Meyer et all, 2012).

Nossos dados apontam um grande número de estudantes na fase de exaustão quando comparado a outros estudos semelhantes (Aguiar, Vieira, Vieira, Aguiar, \& Nóbrega, 2009). Ao final do semestre letivo, 32,7\% dos estudantes se mostram na 
fase de exaustão. Este fato é bastante preocupante pois, esta fase mais avançada do estresse está relacionada a falha nos mecanismos protetores frente aos estímulos estressores e pode tornar os indivíduos vulneráveis a infecções e doenças, e trazendo reações somáticas resultantes da hiperatividade da glândula adrenal.

Dentro da interação do organismo físico com o seu ambiente e a partir da sua interação social e seu impacto psicológico, observa-se uma relação dialética entre o bem-estar e a doença. Isto se deve à busca do equilíbrio, visando o estado de homeostasia do ser humano consigo mesmo e também com seu ambiente externo (Garcia, 2014). O ser humano é o constante resultado atual e histórico de suas próprias interações e construções. Biologicamente a interação física, psicológica e social do ser humano promove diversas ações adaptativas, cuja finalidade é propiciar respostas fisiológicas que permitam este organismo a manutenção do seu estado de sobrevivência. Nosso trabalho mostra um claro aumento na prevalência de estresse bem como piora de seus níveis classificatórios ao final do semestre letivo com sugestão de alterações somáticas aqui representadas pela elevação dos níveis pressóricos.

\section{CONSIDERAÇÕES FINAIS}

Este trabalho propôs a identificação e quantificação do estresse de estudantes universitários de diversas áreas em dois momentos específicos do curso (início e final do semestre letivo). Como ferramenta complementar, monitoramos também a variação da pressão arterial que tem se mostrado como um dos indicadores de alterações psicossomáticas relacionadas a alterações do nível de estresse.

A prevalência de estresse entre os estudantes universitários mostrou uma forte tendência de aumento ao final do semestre letivo. Os estudantes da área de ciências humanas mostraram também uma piora no nível do estresse próximo ao encerramento das atividades acadêmicas semestrais. A maioria dos estudantes avaliados apresentou elevação dos níveis pressóricos ao longo do semestre podendo este fator estar associado ao aumento na prevalência e severidade do estresse. A piora na qualidade de vida ao final do semestre letivo parece ser um fator independente e não relacionado ao incremento do estresse na maior parte população estudada.

Procuramos homogeneizar nossa amostragem trabalhando com uma representatividade de cerca de $5 \%$ dos alunos do Centro Universitário para cada área. No entanto algumas áreas, como a de ciências biológicas e a de cursos com formação tecnóloga apresentam uma amostragem bem menor de estudantes quando comparados a outras áreas, como a área de ciências humanas. Desta forma, para alcançar a representatividade alguns grupos ficaram com um número reduzido de participantes e outros com um número bem maior de representantes. É importante salientar que esta diferença pode diminuir a confiabilidade das comparações entre os grupos. 
Os resultados obtidos neste estudo devem abrir caminho para discussão em relação a metodologias de ensino e avaliação do aprendizado com menor impacto sobre a saúde mental dos estudantes universitários e também ressalta a importância do oferecimento de programas de apoio à saúde mental para esta população.

\section{REFERÊNCIAS}

Adewuya, A. O., Ola, A. B., Aloba, O. O. Mapayi, B. M., \& Oginni, O. O. (2006). Depression amongst nigerian university students: prevalence and sociodemographic correlates. Social psychiatriy and psychiatry epidemiology, 41(8), 674-678. doi:10.1007/s00127-006-0068-9

Agorastos, A., Pervanidou, P., Chrousos, G. P., \& Baker, D. G. (2019). Developmental trajectories of early life stress and trauma: A narrative review on neurobiological aspects beyond stress system dysregulation. Frontiers in Psychiatry, 10, Article 118. doi:10.3389/fpsyt.2019.00118

Aguiar, S. M., Vieira, A. P. G. F., Vieira, K. M. F., Aguiar, S. M., \& Nóbrega, J. O. (2009). Prevalência de sintomas de estresse nos estudantes de medicina. Jornal Brasileiro de Psiquiatria, 58(1), 34 38. doi:10.1590/S0047-20852009000100005.

Bittencourt, A. M., Gottlieb, O. R., Mageswaram, S., Mors, W. B., Ollis, W. D., Sutherland, I. O., \& Magalhaes, M. T. (1971). The natural occurrence of 6-styryl-2-pyrones and their synthesis. Tetrahedron, 27(5), 1043-1048. doi:10.1016/S0040-4020(01)92506-2

Bujang, M. A., Musa, R., Liu, W. J., Chew, T. F., Lim, C. T., \& Morad, Z. (2015). Depression, anxiety and stress among patients with dialysis and the association with quality of life. Asian Journal Psychiatry, 18, 49-52. doi:10.1016/j.ajp.2015.10.004

Cardoso, J. V., Gomes, C. F. M., Junior, R. J. P., \& Silva, D. A. d. (2019). Stress in university students: epidemiological approach. Journal of Nursing - UFPE On Line, 13, 6. doi:10.5205/19818963.2019.241547

Damião Neto, A., Lucchetti, A. L. G., da Silva Ezequiel, O., \& Lucchetti, G. (2019). Effects of a Required Large-Group Mindfulness Meditation Course on First-Year Medical Students' Mental Health and Quality of Life: a Randomized Controlled Trial. Journal General Internal Medicine, 35(3):672-678. doi:10.1007/s11606-019-05284-0

Garcia, M. R. (2014). Origem e evolução: o estresse como resposta adaptativa no contexto da vida moderna. Avesso do Avesso 12(12), 7-15.

Graham, J. D. P. (1945). High blood-pressure after battle. The Lancet, 245, 239-240. doi:10.1016/S01406736(45)91458-9

Group, T. W. (1995). The World Health Organization quality of life assessment (WHOQOL): Position paper from the World Health Organization. Social Science \& Medicine, 41(10), 1403-9. doi: 10.1016/0277-9536(95)00112-k

Ibrahim, A. K., Kelly, S. J., Adams, C. E., \& Glazebrook, C. (2013). A systematic review of studies of depression prevalence in university students. Journal Psychiatry Res, 47(3), 391-400. doi:10.1016/j.jpsychires.2012.11.015

Kluthcovsky, A. C. G. C., \& Kluthcovsky, F. A. (2009). O WHOQOL-bref, um instrumento para avaliar qualidade de vida: uma revisão sistemática. Revista de Psiquiatria do Rio Grande do Sul, 31(3 suppl), 0-0. doi:10.1590/S0101-81082009000400007

Lipp, M. E. N. (1984). Estresse e suas implicações. Estudos de Psicologia, 1(3), 14.

Lipp, M. E. N., \& Guevara, A. J. d. H. (1994). Validação empírica do Inventário de Sintomas de Estresse (ISS). Estudos de Psicologia, 11(3), 8. 
Lipp, M. E. N., Pereira, M. M., Justo, A. P., \& Matos, T. M. De. (2006). Cardiovascular reactivity in hypertensives: differential effect of expressing and inhibiting emotions during moments of interpersonal stress. Spanish Journal Psychology, 9(2), 154-161. doi:10.1017/s1138741600006053

Matthews, K. A., Katholi, C. R., McCreath, H., Whooley, M. A., Williams, D. R., Zhu, S., \& Markovitz, J. H. (2004). Blood pressure reactivity to psychological stress predicts hypertension in the CARDIA study. Circulation, 110(1), 74-78. doi:10.1161/01.CIR.0000133415.37578.E4

Meyer, C., Guimarães, A. C. d. A., Machado, Z., \& Parcias, S. R. (2012). Qualidade de vida e estresse ocupacional em estudantes de medicina. Revista Brasileira de Educação Médica, 36(4), 489498. doi:10.1590/S0100-55022012000600007

Mortier, P., Auerbach, R. P., Alonso, J., Axinn, W. G., Cuijpers, P., Ebert, D. D., Bruffaerts, R. (2018). Suicidal thoughts and behaviors among college students and same-aged peers: results from the World Health Organization World Mental Health Surveys. Society Psychiatry Epidemiology, 53(3), 279-288. doi:10.1007/s00127-018-1481-6

O'Brien, E., Atkins, N., Stergiou, G., Karpettas, N., Parati, G., Asmar, R., . . Hypertension, W. G. o. B. P. M. o. t. E. S. o. (2010). European Society of Hypertension International Protocol revision 2010 for the validation of blood pressure measuring devices in adults. Blood Pression Monitory, 15(1), 23-38. doi:10.1097/MBP.0b013e3283360e98

Ribeiro, Í., Pereira, R., Vidal Freire, I., Oliveira, B., Casotti, C., \& Boery, E. (2017). Stress and Quality of Life Among University Students: A Systematic Literature Review. Health Professions Education, 4(2). doi:10.1016/j.hpe.2017.03.002

Rossetti, M. O., Ehlers, D. M., Guntert, I. B., Leme, I. F. A. d. S., Rabelo, I. S. A., Tosi, S. M. V. D., Barrionuevo, V. L. (2008). O inventário de sintomas de estresse para adultos de lipp (ISSL) em servidores da polícia federal de São Paulo. Revista Brasileira de Terapias Cognitivas, 4(2), 108-120.

Russell, K., Allan, S., Beattie, L., Bohan, J., MacMahon, K., \& Rasmussen, S. (2019). Sleep problem, suicide and self-harm in university students: A systematic review. Sleep Medicine Rev, 44, 58-69. doi:10.1016/j.smrv.2018.12.008

Sadir, M. A., Bignotto, M. M., \& Lipp, M. E. N. (2010). Stress e qualidade de vida: influência de algumas variáveis pessoais. Paidéia (Ribeirão Preto), 20(45), 73-81. doi:10.1590/S0103 863X2010000100010

Saleh, D., Camart, N., \& Romo, L. (2017). Predictors of Stress in College Students. FrontiersinPsychology, 8, 19. doi:10.3389/fpsyg.2017.00019

Selye, H. (1959). Estresse, a tensão da vida. Ibrasa - Instituição Brasileira de Difusão Cultural. São Paulo.

Sheffield, D., Smith, G. D., Carroll, D., Shipley, M. J., \& Marmot, M. G. (1997). The effects of blood pressure resting level and lability on cardiovascular reactions to laboratory stress. Internal Journal Psychophysiol, 27(2), 79-86. doi:10.1016/s0167-8760(97)00044-5

Stearns, S. C., \& Hoekstra, R. F. (2003). Evolução: uma introdução (1 ed.). Atheneu. São Paulo.

Valentino, R. J., \& Van Bockstaele, E. (2008). Convergent regulation of locus coeruleus activity as an adaptive response to stress. European Journal Pharmacolology 583(2-3), 194-203. doi:10.1016/j.ejphar.2007.11.062

Wood, S. K., \& Valentino, R. J. (2017). The brain norepinephrine system, stress and cardiovascular vulnerability. Neuroscience Biobehavyorism Rev, $74(\mathrm{Pt} \quad$ B), $393-400$ doi:10.1016/j.neubiorev.2016.04.018

Yaribeygi, H., Panahi, Y., Sahraei, H., Johnston, T. P., \& Sahebkar, A. (2017). The impact of stress on body function: A review. EXCLI Journal, 16, 1057-1072. doi:10.17179/excli2017-480 


\section{CONFLITOS DE INTERESSE}

Os autores declaram não haver conflito de interesse no trabalho apresentado

\section{SOBRE OS AUTORES}

Luís Henrique Novais é Psicólogo e Mestre em Ciências da Saúde pela Faculdade de Ciências Médicas de Minas Gerais.

e-mail: interpsy2001@yahoo.com.br

(2) https://orcid.org/0000-0002-9057-3368

Bruno Almeida Rezende é Doutor em Fisiologia e Farmacologia e Professor do Programa de Mestrado em Ciências da Saúde da Faculdade de Ciências Médicas de Minas Gerais.

e-mail: bruno.rezende@cienciasmedicasmg.edu.br

(1) https://orcid.org/ 0000-0002-4460-0572 